\title{
Novel In Situ Collection of Tumor Interstitial Fluid from a Head and Neck Squamous Carcinoma Reveals a Unique Proteome with Diagnostic Potential
}

\author{
Matthew D. Stone • Rick M. Odland • Thomas McGowan • Getiria Onsongo • \\ Chaunning Tang • Nelson L. Rhodus • Pratik Jagtap • Sricharan Bandhakavi • \\ Timothy J. Griffin
}

Published online: 25 July 2010

(C) The Author(s) 2010. This article is published with open access at Springerlink.com

\begin{abstract}
Introduction Tumors lack normal drainage of secreted fluids and consequently build up tumor interstitial fluid (TIF). Unlike other bodily fluids, TIF likely contains a high proportion of tumor-specific proteins with potential as biomarkers.

Methods Here, we evaluated a novel technique using a unique ultrafiltration catheter for in situ collection of TIF and used it to generate the first catalog of TIF proteins from a head and neck squamous cell carcinoma (HNSCC). To maximize proteomic coverage, TIF was immunodepleted for high abundance proteins and digested with trypsin, and peptides were fractionated in three dimensions prior to mass spectrometry.

Results We identified 525 proteins with high confidence.
\end{abstract} The HNSCC TIF proteome was distinct compared to

Electronic supplementary material The online version of this article (doi:10.1007/s12014-010-9050-3) contains supplementary material, which is available to authorized users.

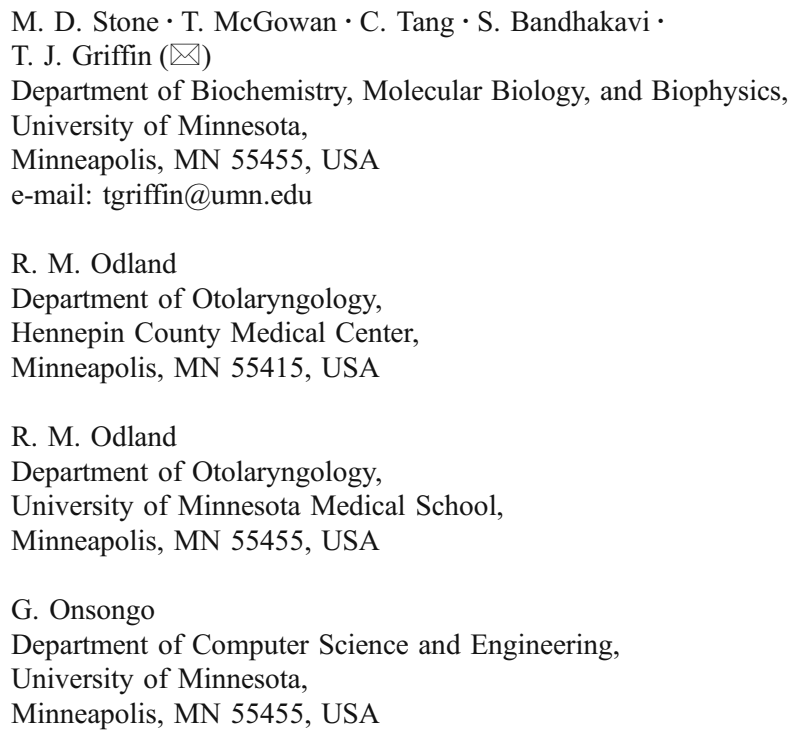

proteomes of other bodily fluids. It contained a relatively high proportion of proteins annotated by Gene Ontology as "extracellular" compared to other secreted fluid and cellular proteomes, indicating minimal cell lysis from our in situ collection technique. Several proteins identified are putative biomarkers of HNSCC, supporting our catalog's value as a source of potential biomarkers.

Conclusions In all, we demonstrate a reliable new technique for in situ TIF collection and provide the first HNSCC TIF protein catalog with value as a guide for others seeking to develop tumor biomarkers.

Keywords Biomarker discovery ·

Head and neck squamous cell carcinoma .

In situ collection - Tumor interstitial fluid

C. Tang

Key Laboratory for Cell Proliferation and Regulation Biology,

Ministry of Education, Beijing Normal University,

Beijing 100875, China

N. L. Rhodus

Department of Oral Medicine, Diagnosis and Radiology,

School of Dentistry, University of Minnesota,

Minneapolis, MN 55455, USA

P. Jagtap

Minnesota Supercomputing Institute, University of Minnesota, Minneapolis, MN 55455, USA 


\section{Introduction}

Squamous cell carcinoma (SCC) is a common type of cancer originating in epithelial cells. Survival rates of SCCs of the upper respiratory tract 5 years post-diagnosis are about $50 \%$ [1]. However, early detection and treatment results in substantially better prognosis [1]. Currently, the most accurate diagnostic test for all SCCs is a histological examination of a tissue biopsy. A less invasive test that still maintains sensitivity and selectivity, from a readily available bodily fluid like plasma or saliva, would be greatly beneficial.

One potential test would be based on specific differential protein expression, or protein biomarkers of SCC. A promising source of possible biomarkers of SCC is tumor interstitial fluid (TIF). The production of TIF results from buildup of fluid derived from cell secretions due to a lack of a fully formed vascular and lymphatic system within the tumor [2]. Because this fluid is directly associated with the tumor, it may be a rich source of tumor-specific biomarkers. In the case of SCC of the head and neck (and potentially SCC at other anatomical locations), TIF may infiltrate other easily sampled bio-fluids such as plasma or saliva. Thus, knowledge of TIF proteomes may help to prioritize putative protein biomarkers found in plasma or saliva to guide validation studies.

Despite its potential value as a source of tumor biomarkers, representative catalogs of TIF proteomes are lacking. Contributing to this lack is the inherent difficulty in effectively extracting TIF directly from human subjects. Currently, a couple of different analysis strategies for studying interstitial fluid have been employed. First, some studies have used indirect extraction of TIF from biopsies where resected tissue is soaked in buffer and proteins diffused into the buffer over time are analyzed [3, 4]. Other studies have used cell culture models to study TIF-like proteomes where conditioned media are collected after a period of incubation and shed/secreted proteins are analyzed [5, 6]. These collections may not be true representations of TIF due to the ex vivo and in vitro sampling techniques employed.

In this study, TIF from a head and neck SCC tumor was obtained directly in situ without biopsy using a novel ultrafiltration catheter, and its proteome was characterized via immunodepletion, trypsin digestion, three-dimensional peptide fractionation, and mass spectrometry. This represents the first study of TIF collected in situ and the first study of TIF obtained from head and neck squamous cell carcinoma (HNSCC). Our collection technique, sample preparation, and resulting proteomic catalog should be a valuable resource to others investigating tumor progression mechanisms as well as those seeking to identify tumor-specific biomarkers of SCC.

\section{Experimental Methods}

In Situ SCC TIF Collection TIF was collected from the tumor of a patient with stage IV head and neck cancer with informed consent using an ultrafiltration catheter previously described [7]. Briefly, the catheter is constructed with hollow fibers composed of polysulfone with $0.45-\mu \mathrm{m}$ pores throughout the walls of the fiber. This allows fluids and prevents solid material from passing through. It is slightly smaller than a 27 -gauge needle, and the proximal shaft of the catheter is the size of a 24-gauge needle to limit disturbances from seeding. The proximal shaft is connected to vacuum allowing for the extraction of fluid. TIF was collected from the right and left side of the tumor, was mixed with phosphate buffered saline, and was immediately stored at $-80^{\circ} \mathrm{C}$. Protein amount was determined from BCA assay (Pierce, Rockford, IL).

Depletion of High Abundance Proteins from TIF To remove high abundance proteins from TIF for enhancement of proteomic coverage, a Multiple Affinity Removal System (MARS, Agilent Technologies, Inc., Palo Alto, CA) column was used. The MARS column consists of immobilized antibodies directed against albumin, $\operatorname{IgG}, \operatorname{IgA}$, transferrin, haptoglobin, and antitrypsin. Protein from TIF (0.4 mg) was applied to the MARS column, and the column was used according to manufacturer's instructions using manufacturer-supplied buffers. Fractions consisting of flow-through of unbound and eluted bound proteins were collected separately. The flow-through fraction contained $0.15 \mathrm{mg}$ of protein.

Trypsin Digestion and Fractionation of TIF Peptides Both undepleted $(100 \mu \mathrm{g})$ and MARS-depleted $(150 \mu \mathrm{g})$ TIF protein samples were independently digested and used for proteomics analysis. As a denaturant, SDS was added to TIF samples to a final concentration of $0.5 \% \mathrm{w} / \mathrm{v}$. Dithiothreitol (Sigma) was added at $10 \mathrm{mM}$ and incubated for $60 \mathrm{~min}$ at $56^{\circ} \mathrm{C}$. Resulting free sulfhydryl groups were alkylated with addition of $50 \mathrm{mM}$ iodoacetamide (Sigma) for $45 \mathrm{~min}$ at room temperature in the dark. Unreacted iodoacetamide was neutralized with further addition of $40 \mathrm{mM}$ dithiothreitol. Samples were diluted tenfold with $10 \mathrm{mM}$ ammonium bicarbonate buffer $(\mathrm{pH} \mathrm{8.0)}$ to reduce the SDS concentration to $0.05 \% w / v$. Sequencing grade trypsin (Promega, Madison, WI) was added to a final enzyme/substrate ratio of 1:50, and the reaction was incubated at $37^{\circ} \mathrm{C}$ overnight. The next day, samples were 
cleaned with an Oasis ${ }^{\circledR}$ MCX solid phase extraction cartridge (Waters, Milford, MA) according to manufacturer's protocol, and the eluted peptides were dried by vacuum centrifugation.

Peptide isoelectric focusing (IEF) was performed using an Agilent 3100 OFFGEL Fractionator (Agilent) [8] using the 24-well setup with $\mathrm{pH}$ 3-10 IPG strips according to manufacturer's instructions. Briefly, peptides resulting from trypsin digestion of TIF proteins were dissolved in $3.6 \mathrm{~mL}$ of OFFGEL peptide sample solution consisting of appropriate ampholytes. The IPG strip was rehydrated with the Peptide IPG Strip Rehydration Solution for $15 \mathrm{~min}$. The peptide solutions (either from the non-depleted or depleted sample) were separately loaded in equal volumes $(150 \mu \mathrm{L})$ into each of the wells. The peptides were then electrofocused at a maximum limit of $50 \mu \mathrm{A}$ until $50 \mathrm{kVh}$ was reached. After completion, the peptide IEF fractions from individual wells were collected. Peptide IEF fractions from the non-depleted samples were acidified to $\mathrm{pH} \sim 2$ with TFA, purified using "Stage" tips [9] composed of $2 \times 22$-gauge needle punches of Empore ${ }^{\mathrm{TM}}$ extraction disks ( $3 \mathrm{M}$, St. Paul, MN), dried by vacuum centrifugation, and stored pending mass spectrometric analysis. The MARS depleted samples were further fractionated with offline strong cation exchange (SCX) HPLC as described previously [10].

Capillary LC-MS/MS Dried peptides were resuspended in $5 \mu \mathrm{L}$ of load buffer (98:2:0.1 water/acetonitrile/formic acid). Theses samples were loaded onto an in-house poured reversed phase capillary column at $1,000 \mathrm{~nL} / \mathrm{min}$ using an Eksigent MicroAS autosampler and 1DLC nano-HPLC. The column consisted of fused silica tubing packed with Magic $\mathrm{C} 18 \mathrm{AQ}-5-\mu \mathrm{m}, 200-\AA$ pore size resin (Michrom Bioresources) to dimensions of $13 \mathrm{~cm} \times 100 \mu \mathrm{m}$, and the outlet of the column was a pulled tip. Reversed phase chromatography followed a gradient of $2-40 \%$ acetonitrile in $0.1 \%$ formic acid over $90 \mathrm{~min}$ at $250 \mathrm{~nL} / \mathrm{min}$. The tip of the column was in line with an LTQ-Orbitrap XL mass spectrometer (ThermoFisher Scientific). Eluting solvent was ionized using a nano-electrospray source. Spray voltage was set to $1.75 \mathrm{kV}$, and the capillary ion transfer tube was set to $160^{\circ} \mathrm{C}$. Mass spectrometry was performed using gas-phase fractionation as described elsewhere [11]. A series of four separate full scans were acquired from the orbital trap $(m / z 380-520 ; 515-690 ; 685-975 ; 970-2,000)$ at a resolution of 60,000 at $\mathrm{m} / \mathrm{z} 400$ with AGC settings of $1 \mathrm{E} 6$ ions or $500 \mathrm{~ms}$. The top three intense ions from each individual full scan fraction were subjected to fragmentation by CID at a normalized collision energy of 35\% and scanned out using the LTQ ion trap. AGC settings for the LTQ ion trap were 1E4 ions or $100 \mathrm{~ms}$. Charge state screening was enabled for full scans so that unassigned charge states and singly charged ions were rejected for CID. Ions previously selected for CID were also excluded from further analysis using dynamic exclusion. The exclusion list was set to the maximum default value of 500 entries. Exclusion time was set to $60 \mathrm{~s}$ with a mass tolerance window of -0.6 to $1.2 \mathrm{amu}$. Spectral data were acquired and saved using Xcalibur software.

Cheek Cell Brush Biopsy Collection and Analysis The inside of the cheek of a healthy volunteer was dried with a cotton swab to minimize salivary contamination, and buccal epithelial cells were obtained with brushing using an OralCDx brush test kit (OralCDx Laboratories, Inc., Suffern, NY). The inner cheek was brushed ten times in a consistent direction. After that, the oral brush was rotated $360^{\circ}$ for three times on the tissue surface. The applied pressure was just enough to slightly bow the handle of the brush. The brush head was placed in $0.25 \mathrm{~mL}$ of lysis buffer (4\% SDS, 10\% 2-mercaptoethanol, $100 \mathrm{mM}$ Tris$\mathrm{HCl}, \mathrm{pH} 6.8$, and $1 \times$ protease inhibitors (Complete Mini, Roche Applied Science, Indianapolis, IN, USA)), and the exfoliated epithelial cells were lysed with continuous vortexing for $15 \mathrm{~min}$ at room temperature. The protein lysate $(0.25 \mathrm{~mL})$ was chilled on ice prior to addition of $1 \mathrm{~mL}$ of ice-cold acetone to precipitate proteins overnight at $-20^{\circ} \mathrm{C}$. Precipitated protein was centrifuged at $6,000 \mathrm{rpm}$ for $10 \mathrm{~min}$ at $4^{\circ} \mathrm{C}$, and the protein pellet was dissolved in $50 \mathrm{mM}$ Tris- $\mathrm{HCl}$, pH 8.5 containing $5 \mathrm{mM}$ EDTA. An aliquot containing $0.1 \mathrm{mg}$ of protein was digested with sequencing grade trypsin (Promega, Madison, WI) at a 1:100 enzyme/substrate ratio. Digestion occurred overnight at $37^{\circ} \mathrm{C}$, and resulting peptides were subjected to SCX as described above. A total of nine fractions were analyzed by reversed phase nLC MS/MS essentially as described [10]

Database Searching and Data Processing Mass spectral data were searched using MaxQuant software [12]. MSM files were generated using the Quant feature. Parameters included full trypsin specificity with up to two missed cleavages and oxidized methionine as a variable modification. The TIF data also included Cys alkylation as a fixed modification. The MSM files were searched against a composite of the IPI human database v3.52 and its reversed compliment including common contaminant proteins using Mascot [13] with $7 \mathrm{ppm}$ precursor and 0.5 amu fragment ion mass tolerances, respectively. Identification parameters included minimum PEP score of 1, at least two unique peptides for identification, and protein false discovery rate (FDR) $<1 \%$.

Comparison of TIF to Other Proteomes The salivary protein dataset was generated from .raw files obtained from [10] and was searched using MaxQuant and parameters as 
above. It contained 2,096 proteins identified from two or more unique peptide sequences. Plasma proteins were obtained from publicly available Human Plasma Proteome Project, http://www.bioinformatics.med.umich.edu/hupo/ ppp. This dataset contained 3,020 proteins which were identified with at least two unique peptides. Protein datasets from conditioned media of the ovarian cancer cell lines: HTB-75, RMUG-S, TOV-112D, and TOV-21G were obtained from [5]. Identifications were filtered based on $95 \%$ peptide probability and $80 \%$ protein probability for PeptideProphet [14] and ProteinProphet [15], respectively. The liver interstitial fluid (IF) proteomes were from [4], and each identification had at least two unique peptide matches. Proteomic comparisons were accomplished through matching of IPI accession numbers.

Comparison Using Gene Ontology Terms Proteins identified from TIF and epithelial brush biopsy cell lysate were compared by the cellular components Gene Ontology (GO) term. The resulting MaxQuant identification output contained Gene Ontology names corresponding to each protein. Names that included "cytosolic," "cytoplasmic," or "cytoskeletol" were counted and grouped as "cytoplasmic." Names that referred to organelles such as the endoplasmic reticulum, the nucleus, mitochondria, the Golgi complex, peroxisomes, or lysosomes were counted and grouped as "organellar." Proteins without term information or with no apparent location were grouped as "unclassified." Several proteins grouped into multiple categories.

\section{Results}

In Situ Collection of TIF For our study, a unique ultrafiltration catheter [7, 16] was used for in situ TIF collection from a patient with a stage IV neck SCC. The catheter, originally designed to deliver therapeutics to localized sites, was composed of a fibrous wall 3-mm long with an outer diameter of $380 \mu \mathrm{m}$ similar to that of a 28 -gauge needle. The walls were made of semi-permeable hydrophilic polysulfone fiber with $0.45-\mu \mathrm{m}$ pores to prevent solid material from passing through. The porous fiber also had a relatively large surface area to dissipate the force generated from suction, thereby providing gentle extraction. In all, the miniature design with large surface area was intended to limit tissue damage. The distal end was enclosed, and the proximal end was connected to a Vacutainer, providing suction for removal and storage of the TIF.

Sample Preparation and Proteomic Analysis of TIF TIF was collected from two bilateral regional metastases of a stage IV HNSCC in a single patient with informed consent.
The extracted fluids were mixed with phosphate-buffered saline and immediately stored at $-80^{\circ} \mathrm{C}$ until further use. The total protein amount extracted was $0.6 \mathrm{mg}$ according to BCA assay. The Coomassie staining pattern after SDSPAGE showed that the two TIF collections were very similar (Fig. 1, lanes 1 and 2), suggesting that the TIF was homogenous throughout the tumor and that extraction was consistent and repeatable. The detection of only a few intense bands by Coomassie staining indicated the possible presence of several proteins at much higher abundance compared to other proteins in the sample, similar to the large dynamic range of protein abundances observed in other bio-fluids like plasma.

To get an initial assessment of the protein composition of TIF, a small portion was digested with trypsin, and the peptides were analyzed via tandem mass spectrometry (MS/MS). The seven identified proteins that had the highest number of assigned spectra (starting with the highest in descending order) were albumin, hemoglobin (beta and alpha subunits), transferrin, complement component 3 precursor, haptoglobin isoform 2 preprotein, alpha-2-macroglobulin, and alpha-1-antitrypsin. Consistent with these results, the most prominent bands in Fig. 1 at $\sim 70$ and $\sim 15 \mathrm{kDa}$ match the expected molecular weight of albumin and alpha/beta globin, respectively. Except for the two hemoglobin subunits, the other six proteins are among the most abundant proteins found in plasma $[17,18]$.

Seeking to expand our identification of lower abundance proteins, we chose to remove the high abundance proteins through immunodepletion using a MARS column designed to remove the abundant plasma proteins we found in TIF. After starting with $\sim 0.4 \mathrm{mg}$, the depleted flow-through from

Fig. 1 SDS-PAGE analysis of TIF. TIF $(10 \mu \mathrm{g})$ was separated by SDS-PAGE and stained with Coomassie brilliant blue. The two lanes labeled right and left represent TIF collected from two separate extractions using the ultrafiltration catheter device of a head and neck tumor in situ

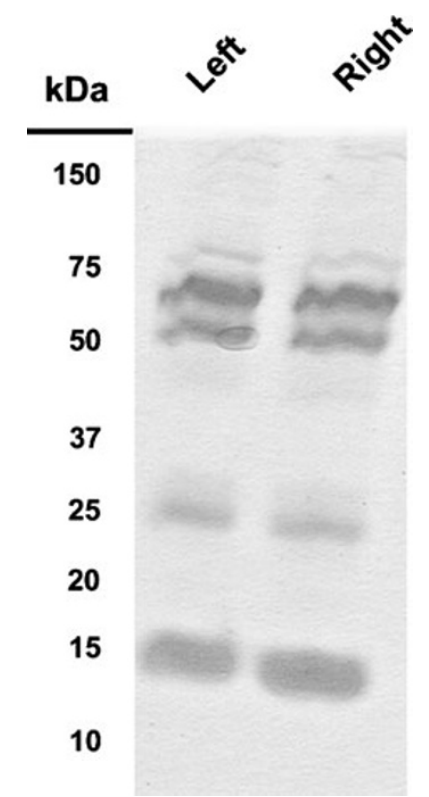


the MARS column contained $0.15 \mathrm{mg}$ of total protein. These proteins were digested with trypsin, and the peptides were separated based on isoelectric point. We then incorporated SCX chromatography as depicted in Fig. 2, which outlines the sample preparation and analysis scheme. SCX had previously been shown to be an effective second fractionation step after isoelectric focusing for complex samples $[10,19]$. Specifically, the isoelectric point peptide fractions were combined in groups of six to eight fractions creating three separate pools, each of which was subjected to SCX HPLC. A total of 12-15 fractions from each pooled sample were selected based on signal from UV chromatograms of the associated SCX fractionation [10]. Each of these fractions was individually subjected to $\mu \mathrm{LC}-\mathrm{MS} / \mathrm{MS}$ analysis, which resulted in identification of 525 proteins with at least two unique peptide sequences. The global protein level FDR was $<1 \%$. All of the identified peptides are listed in Supplementary Table 1, and the corresponding proteins are listed in Supplementary Table 2.

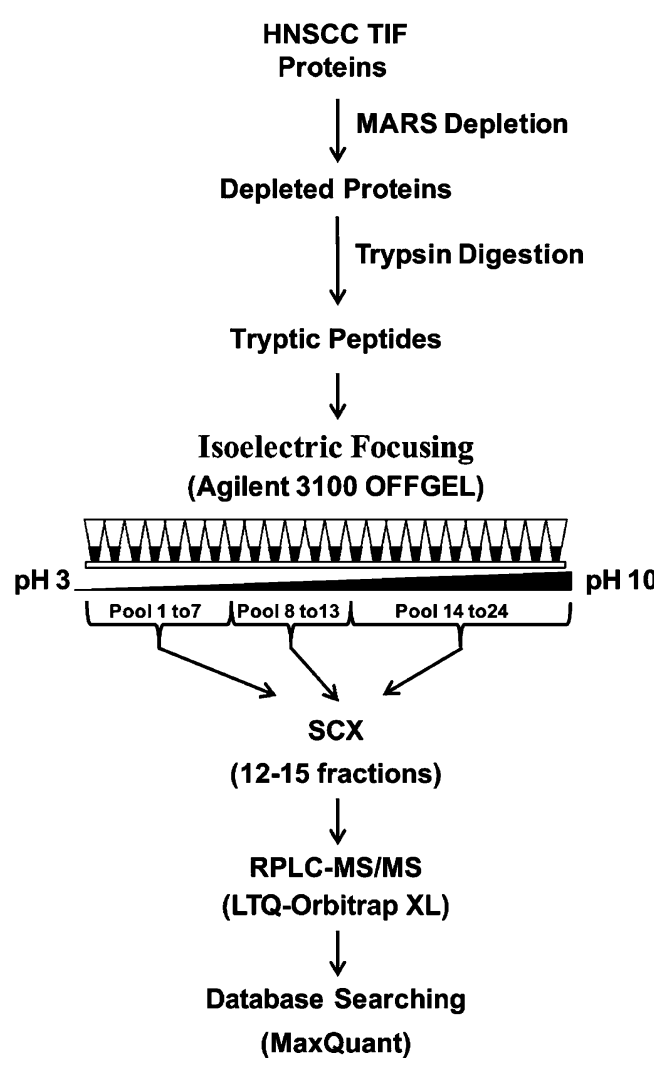

Fig. 2 Proteomic analysis strategy for identifying proteins from TIF. High abundance proteins in TIF were removed using a MARS column. Remaining proteins were digested with trypsin to generate peptides. The peptides were subjected to three dimensions of fractionation using isoelectric focusing, strong cation exchange chromatography, and reversed-phase chromatography prior to tandem mass spectrometry analysis. MS/MS data was searched against a human database using MaxQuant
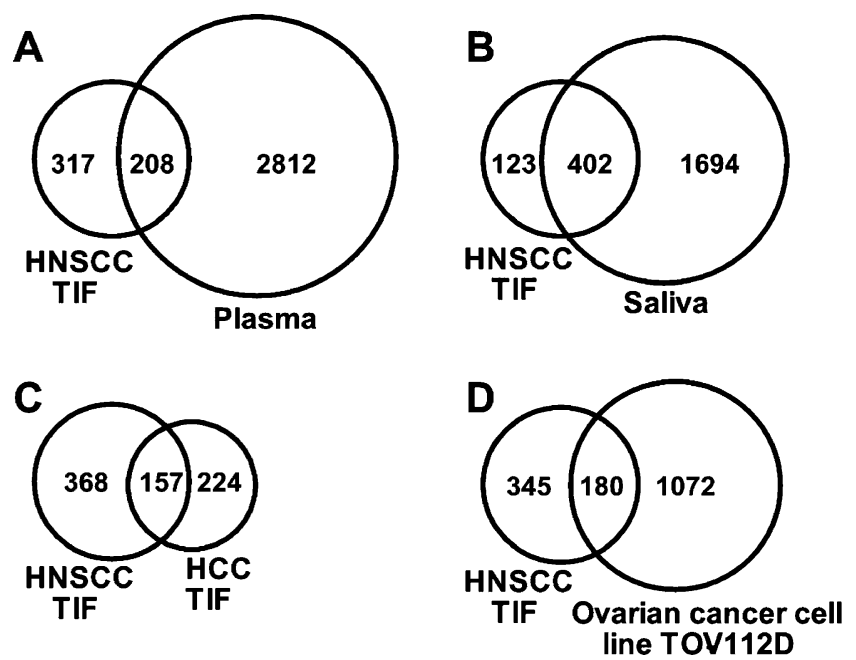

Fig. 3 Comparison of proteins identified in TIF to other proteomes. Venn diagrams showing common and unique proteins from HNSCC TIF to a plasma, $40 \%$ in common; b saliva, $76 \%$ in common; c hepatocyte cell carcinoma TIF, $41 \%$ in common; and $\mathbf{d}$ conditioned media from TOV-112D ovarian carcinoma cell line, 34\% in common

Comparison of HNSCC TIF to Other Proteomes With a novel, high confidence protein catalog in hand, we next sought to compare it with other proteomes to further characterize the HNSCC TIF proteome. Since HNSCC TIF contained several high abundance plasma proteins, we initially compared it against the plasma proteome to determine the degree of similarity between these two proteomes. The human plasma proteome was obtained from the HUPO Plasma Proteome Project (http://www. bioinformatics.med.umich.edu/hupo/ppp) and contained 3,020 proteins with at least two unique peptide identifications. Unexpectedly, HNSCC TIF had a relatively small overlap of common proteins with plasma $(40 \%$ overlap, Fig. 3a). In fact, HNSCC TIF shared more in common with the proteome of whole saliva $(76 \%$ overlap, Fig. 3b). This finding was even more striking considering that the plasma proteome had about 1,000 more proteins than saliva. The relatively low amount of overlap with plasma supported that the HNSCC TIF was collected with little or no accidental contamination from blood. Thus, the ultrafiltration catheter appeared to avoid damage to blood vessels.

We next sought a more direct comparison of the HNSCC TIF proteome to other IF or IF-like proteomes. Recently, the proteomes of liver tissue IFs were reported [4]. The IFs were derived from tissue biopsies and obtained ex vivo from proteins released into buffer. These samples included TIF from a hepatocellular carcinoma (HCC), IF from a nontumor part of the same liver, and IF from a separate healthy liver. Not surprisingly, the HCC TIF proteome had the most in common with the HNSCC TIF proteome with $41 \%$ overlap (Fig. 3c), whereas the IF from a non-tumor part of 
the same liver and the IF from a separate healthy liver had $38 \%$ and $35 \%$ of proteins in common. Even though there was $41 \%$ overlap, the HCC and HNSCC TIF proteomes had large numbers of non-shared proteins suggesting that they were quite distinct.

Finally, the HNSCC TIF was compared to secreted proteins from four different ovarian cancer cell lines. The proteomic datasets were obtained in a previous study [5] where an extensive analysis was performed using conditioned media after incubation with cells in order to obtain shed or secreted proteins, similar in principle to those found in TIF. This type of rigorous proteomics analysis has not been performed for HNSCCbased cell models, and despite differences, an ovarian cancer model seemed appropriate since both cancer types are epithelial in nature. HNSCC TIF had $34 \%$ proteins in common with media from TOV-112D cells (Fig. 3d). There was somewhat less overlap with media from HTB75 cells (28\%), TOV-21G cells (29\%), and RMUG-S (18\%; data not shown). Once again, the HNSCC TIF proteome had a high amount of non-overlapping proteins further demonstrating that our in situ collected HNSCC TIF proteome was distinct.

\section{Cellular Localization of HNSCC TIF Proteins}

The identified HNSCC TIF proteins were classified according to the Gene Ontology term "cellular components" to determine cellular localizations. Proteins associated with the cytoskeleton were grouped in "cytoplasmic," and proteins associated with the endoplasmic reticulum, Golgi complex, mitochondrion, lysosome, peroxisome, or nucleus were grouped as "organellar." In cases of ambiguity, proteins were grouped into more than one category. In order to assess potential cell lysis caused by our in situ collection technique, the cellular localization of the HNSCC TIF proteome (525 proteins) was compared to a similar sized proteome (524 proteins) identified from lysed cells gathered from brushing the cheek buccal epithelium of a healthy volunteer (Fig. 4). If our collection technique caused a significant amount of cell lysis, we expected that the HNSCC TIF proteome would show a similar proportion of cytoplasmic and organellar proteins as compared to the epithelial cell proteome. As expected, the cellular lysate contained a large amount of cytoplasmic (421) and organellar (374) proteins, whereas only 61 proteins were classified as extracellular. On the other hand, HNSCC TIF proteome had lower amounts of proteins classified as cytoplasmic and organellar, 339 and 269, respectively, but a comparatively higher proportion of proteins grouped as extracellular (203 total). The overall percentage of proteins grouped as extracellular for HNSCC TIF was high even

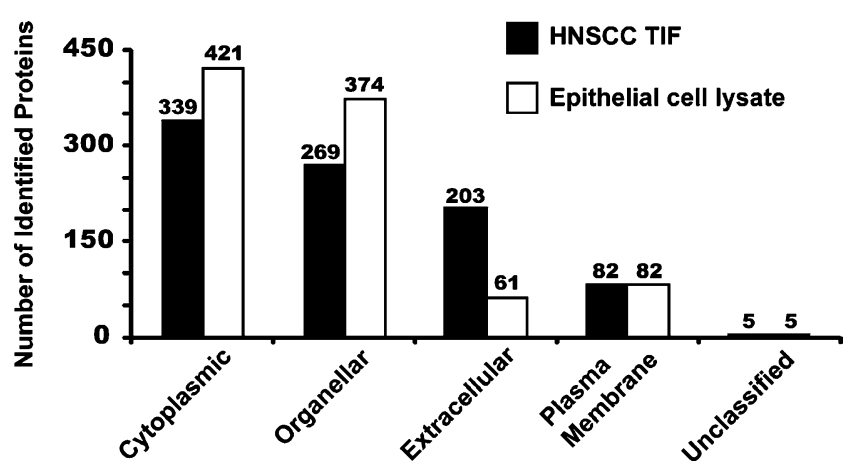

Fig. 4 Comparison of HNSCC TIF and healthy buccal epithelial cell lysate from brush biopsy using the cellular components Gene Ontology term. Identified proteins of HNSCC TIF (solid bars) and epithelial cell lysate (open bars) were categorized into different cellular localizations. Proteins with names associated with cytosol or cytoskeleton were grouped as cytoplasmic. Proteins with localizations for the nucleus, mitochondria, the endoplasmic reticulum, the Golgi complex, peroxisomes, or lysosomes were grouped as organellar. Several proteins were grouped into more than one bin. Proteins with no Gene Ontology information or no clear localization were grouped as unclassified

compared to known extracellular fluids like saliva (data not shown). In all, this data was in agreement with a low level cell lysis associated with the HNSCC TIF sample.

Potential Protein Biomarkers Found in HNSCC TIF The proteins of HNSCC TIF were examined to see if any had been previously reported as a potential biomarker for HNSCC. In a recent review, Schaaij-Visser et al. [20] compiled a list of putative biomarkers for SCC from several different proteomics-based studies. The list, shown in Table 1, was modified to contain biomarkers specific for cancer or cancer progression, which came from in vivo sources of bio-fluid and were up-regulated compared to healthy states. We identified several putative up-regulated protein biomarkers that were identified in HNSCC TIF. Some were identified with several unique peptides and high sequence coverage such as catalase (60\% coverage), S100A9 (56.1\% coverage), and profilin (59.3\% coverage). This suggests that TIF may be a bountiful source of tumorrelated biomarkers.

\section{Conclusions}

This study describes the first report to our knowledge of the proteomic characterization of HNSCC TIF. It is also the first report of proteomic analysis of TIF obtained in situ. Initial characterization showed that HNSCC TIF had a large dynamic range of protein abundance (see Fig. 1). Many of the high abundance proteins were the same high abundance proteins found in plasma. As a result, we chose to use MARS depletion coupled with 


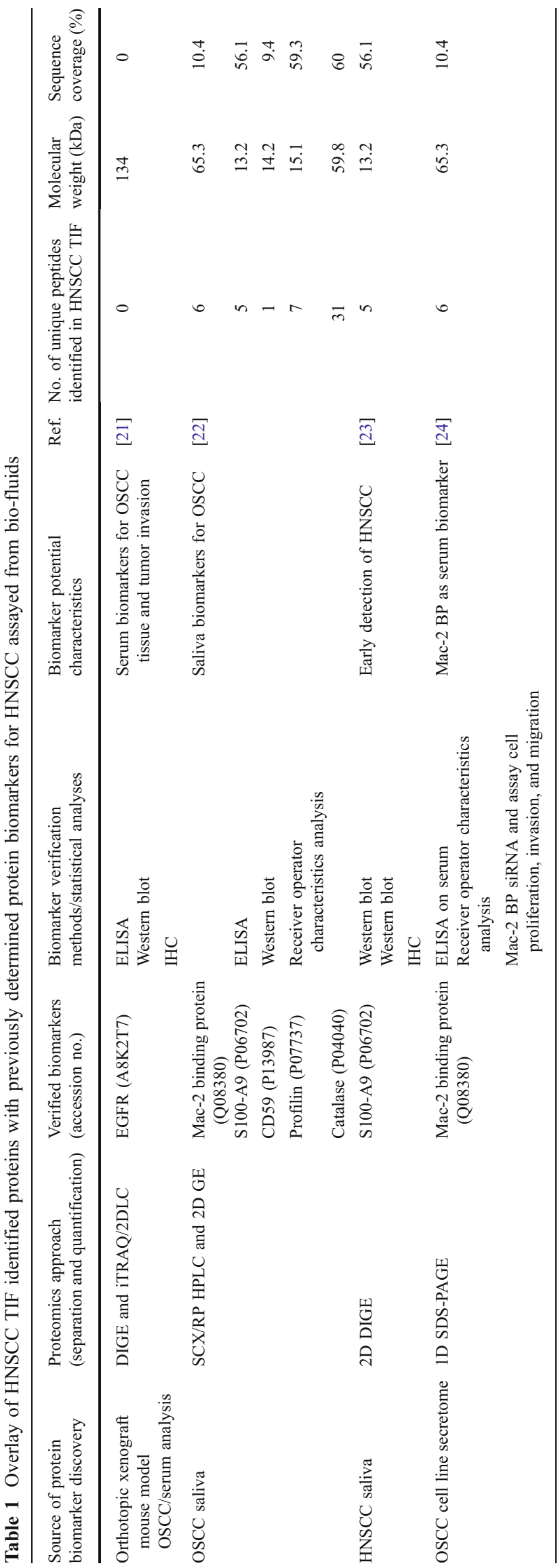

three-dimensional peptide fractionation in our sample preparation, which enabled deeper proteomic coverage. The scheme employed here, as depicted in Fig. 2, may serve as a template for future TIF proteomic studies. In all, 525 proteins were identified with high confidence.

As a whole, our results indicated that the ultrafiltration catheter effectively collected TIF in situ and preserved its integrity by limiting contamination from other sources. First, SDS-PAGE analysis of two separate collections of TIF from the same tumor showed that each collection was consistent. If the samples were contaminated with proteins due to cell lysis or blood vessel damage, we would have expected more random protein patterns detected between the samples. Second, HNSCC TIF identified proteins had a relatively small overlap to proteins identified from plasma (Fig. 3a). This demonstrated that the catheter did not accidentally damage blood vessels leading to introduction of blood proteins into our sample. Third, grouping of protein identifications based on the "cellular components" Gene Ontology term revealed that the TIF proteome had a high proportion of proteins grouped as extracellular and a low proportion of proteins classified as cytoplasmic and organellar compared to a similar sized dataset of an epithelial cell lysate (Fig. 4). The localization of proteins identified from TIF was consistent with that of an extracellular fluid and further suggested that the insertion of the catheter caused minimal cell damage.

The TIF proteome characterized here was quite distinct when compared to other relevant proteomes as shown in Fig. 3. There was a relatively low overlap of identified proteins when compared to interstitial fluids derived from liver and when compared to proteins secreted into conditioned media from various ovarian cancer cell lines. Part of the low overlap is likely due to the different biological sources of the fluids. However, the distinctiveness of the HNSCC TIF is also probably a result of the unique sample collection technique. Previous studies of TIF have relied on excision of tissue biopsies followed by soaking of the extracted tissue over time in buffer. This method is comparably quite destructive and has invariably led to identification of contaminating proteins, which may negatively affect biomarker studies. The unique technique used in this study allowed for gentle collection of TIF in situ, without a biopsy or time delay. Overall, our in situ technique reliably collects high fidelity TIF samples, and it should prove beneficial for further studies characterizing fluid from other types of tumors.

TIF protein catalogs obtained from high fidelity samples, such as the HNSCC catalog presented here, have potential value in prioritization of potential biomarkers found in other studies. There has been a large push in the field of proteomics to find protein biomarkers from easily accessible fluids such as plasma or saliva. Numerous potential biomarkers are typically identified from large-scale quantitative 
proteomics discovery studies. It is difficult to validate all of these potential biomarkers because each validation requires it own specific and sensitive test, which together is rate-limiting. Consequently, prioritization is usually necessary, enabling validation of the most promising potential biomarkers. Given the rich source of potential biomarkers provided by the catalog of TIF proteins, it should serve as a valuable guide to help prioritize potential biomarkers discovered in other bodily fluids for validation. Indeed, a number of proteins identified in our study were previously shown to be up-regulated in SCC progression (Table 1). Thus, our TIF protein catalog should be beneficial for future biomarker studies.

Acknowledgments This research was funded in part by NIH grant 1R01DE017734. We are thankful to Mark Nelson and John Chilton at the University of Minnesota Supercomputing Institute for computational support for database searching. We are thankful to Todd Markowski of the University of Minnesota Proteomics Core Facility for SCX fractionation. We are also thankful to the members of the Griffin laboratory for helpful discussions and comments.

Disclosure Dr. Odland is cofounder and Medical Director of Twin Star Medical, Minneapolis, MN, a company which is developing ultrafiltration catheters for clinical use and supplied the catheters used in this study.

Open Access This article is distributed under the terms of the Creative Commons Attribution Noncommercial License which permits any noncommercial use, distribution, and reproduction in any medium, provided the original author(s) and source are credited.

\section{References}

1. Jemal A, Clegg LX, Ward E, Ries LA, et al. Annual report to the nation on the status of cancer, 1975-2001, with a special feature regarding survival. Cancer. 2004;101:3-27.

2. Baronzio G, Freitas I, Kwaan HC. Tumor microenvironment and hemorheological abnormalities. Semin Thromb Hemost. 2003;29:489-97.

3. Celis JE, Gromov P, Cabezon T, Moreira JM, et al. Proteomic characterization of the interstitial fluid perfusing the breast tumor microenvironment: a novel resource for biomarker and therapeutic target discovery. Mol Cell Proteomics. 2004;3:327-44.

4. Sun W, Ma J, Wu S, Yang D, et al. Characterization of the liver tissue interstitial fluid (TIF) proteome indicates potential for application in liver disease biomarker discovery. J Proteome Res. 2010;9:1020-31.

5. Gunawardana CG, Kuk C, Smith CR, Batruch I, et al. Comprehensive analysis of conditioned media from ovarian cancer cell lines identifies novel candidate markers of epithelial ovarian cancer. J Proteome Res. 2009;8:4705-13.

6. Faca VM, Ventura AP, Fitzgibbon MP, Pereira-Faca SR, et al. Proteomic analysis of ovarian cancer cells reveals dynamic processes of protein secretion and shedding of extra-cellular domains. PLoS ONE. 2008;3:e2425.
7. Oh S, Odland R, Wilson SR, Kroeger KM, et al. Improved distribution of small molecules and viral vectors in the murine brain using a hollow fiber catheter. J Neurosurg. 2007;107:568-77.

8. Horth P, Miller CA, Preckel T, Wenz C. Efficient fractionation and improved protein identification by peptide OFFGEL electrophoresis. Mol Cell Proteomics. 2006;5:1968-74.

9. Rappsilber J, Ishihama Y, Mann M. Stop and go extraction tips for matrix-assisted laser desorption/ionization, nanoelectrospray, and LC/MS sample pretreatment in proteomics. Anal Chem. 2003;75:663-70.

10. Bandhakavi S, Stone MD, Onsongo G, Van Riper SK, Griffin TJ. A dynamic range compression and three-dimensional peptide fractionation analysis platform expands proteome coverage and the diagnostic potential of whole saliva. J Proteome Res. 2009;8:5590-600.

11. Scherl A, Shaffer SA, Taylor GK, Kulasekara HD, et al. Genomespecific gas-phase fractionation strategy for improved shotgun proteomic profiling of proteotypic peptides. Anal Chem. 2008;80: 1182-91.

12. Cox J, Mann M. MaxQuant enables high peptide identification rates, individualized p.p.b.-range mass accuracies and proteomewide protein quantification. Nat Biotechnol. 2008;26:1367-72.

13. Perkins DN, Pappin DJ, Creasy DM, Cottrell JS. Probabilitybased protein identification by searching sequence databases using mass spectrometry data. Electrophoresis. 1999;20:3551-67.

14. Keller A, Nesvizhskii AI, Kolker E, Aebersold R. Empirical statistical model to estimate the accuracy of peptide identifications made by MS/MS and database search. Anal Chem. 2002;74:5383-92.

15. Nesvizhskii AI, Keller A, Kolker E, Aebersold R. A statistical model for identifying proteins by tandem mass spectrometry. Anal Chem. 2003;75:4646-58.

16. Odland RM, Kizziar R, Rheuark D, Simental A. The effect of capillary ultrafiltration probes on skin flap edema. Otolaryngol Head Neck Surg. 2003;128:210-4.

17. Huang L, Harvie G, Feitelson JS, Gramatikoff K, et al. Immunoaffinity separation of plasma proteins by $\operatorname{IgY}$ microbeads: meeting the needs of proteomic sample preparation and analysis. Proteomics. 2005;5:3314-28.

18. Zolotarjova N, Martosella J, Nicol G, Bailey J, et al. Differences among techniques for high-abundant protein depletion. Proteomics. 2005;5:3304-13.

19. Xie H, Onsongo G, Popko J, de Jong EP, et al. Proteomics analysis of cells in whole saliva from oral cancer patients via value-added three-dimensional peptide fractionation and tandem mass spectrometry. Mol Cell Proteomics. 2008;7:486-98.

20. Schaaij-Visser, T. B., Brakenhoff, R. H., Leemans, C. R., Heck, A. J., Slijper, M., Protein biomarker discovery for head and neck cancer. J Proteomics. 2010; (in press).

21. Bijian K, Mlynarek AM, Balys RL, Jie S, et al. Serum proteomic approach for the identification of serum biomarkers contributed by oral squamous cell carcinoma and host tissue microenvironment. J Proteome Res. 2009;8:2173-85.

22. $\mathrm{Hu} \mathrm{S}$, Arellano M, Boontheung $\mathrm{P}$, Wang J, et al. Salivary proteomics for oral cancer biomarker discovery. Clin Cancer Res. 2008;14:6246-52.

23. Dowling P, Wormald R, Meleady P, Henry M, et al. Analysis of the saliva proteome from patients with head and neck squamous cell carcinoma reveals differences in abundance levels of proteins associated with tumour progression and metastasis. J Proteomics. 2008;71:168-75.

24. Weng LP, Wu CC, Hsu BL, Chi LM, et al. Secretome-based identification of Mac-2 binding protein as a potential oral cancer marker involved in cell growth and motility. J Proteome Res. 2008;7:3765-75. 